\title{
Effects of the soy isoflavones, genistein and daidzein, on male rats' skin
}

\author{
Ewa Duchnik¹, Joanna Kruk², Irena Baranowska-Bosiacka³, Anna Pilutin ${ }^{4}$, Romuald Maleszka1 ${ }^{1}$ Mariola Marchlewicz ${ }^{5}$
}

\begin{abstract}
${ }^{1}$ Department of Dermatology and Venereology, Pomeranian Medical University, Szczecin, Poland ${ }^{2}$ Faculty of Physical Culture and Health, University of Szczecin, Szczecin, Poland ${ }^{3}$ Department of Biochemistry and Medical Chemistry, Pomeranian Medical University, Szczecin, Poland ${ }^{4}$ Department of Histology and Embryology, Pomeranian Medical University, Szczecin, Poland ${ }^{5}$ Department of Aesthetic Dermatology, Pomeranian Medical University, Szczecin, Poland
\end{abstract}

Adv Dermatol Allergol 2019; XXXVI (6): 760-766

DOI: https://doi.org/10.5114/ada.2019.87280

\begin{abstract}
Introduction: Genistein and daidzein are typical soy isoflavones with known estrogenic properties to provide protection against skin ageing in postmenopausal women and female rats. However their effect on male skin was very rarely studied.

Aim: This study was designed to evaluate the effect of a mixture of genistein and daidzein on male rats' skin. Material and methods: Male rats were administered this mixture in a dose of 2 or $20 \mathrm{mg} / \mathrm{kg}$ body weight (bw) per day for 5 days weekly mixed with regular rat chow, from prenatal life until sexual maturity. The female and male rats of the control group received regular rat chow. The skin epidermis thickness, number of fibroblasts in the dermis and diameter of collagen fibers in the dermis were measured using morphometric assay. The isoflavone effects on activities of antioxidant enzymes, lipid peroxides, and glutathione concentration in the skin were measured with commercially available kits.

Results: The thickness of the skin epidermis and collagen fibers in the dermis and amount of elastic fibers were significantly greater in the isoflavone-treated groups. Isoflavones significantly decreased catalase activity in the skin homogenates and at a higher dose inhibited lipid peroxides formation.

Conclusions: Our results provide further support for the contribution of isoflavones to defence mechanisms against oxidative stress in the skin and suggest that genistein and daidzein supplementation may provide protection against skin ageing in males.
\end{abstract}

Key words: antioxidant enzymes, collagen, daidzein, genistein, fibroblasts, male rats.

\section{Introduction}

Plant-derived isoflavones are nonsteroidal compounds that exhibit agonistic or antagonistic oestrogen activities, due to their heterocyclic phenolic structure similar to both natural and synthetic oestrogens [1, 2]. Due to their structure similarity to $17 \beta$-estradiol, isoflavones are able to bind oestrogen receptors $E R \alpha$ and $E R \beta$ and to mimic estrogenic actions [3]. Recent data indicated that isoflavones, via binding to $E R \alpha$, promote cell proliferation, but via binding to ER $\beta$, promote apoptosis [4]. Furthermore, they can act as antioxidants by scavenging free radicals and/or chelating metal ions [5, 6]. Isoflavones exhibit anti-inflammatory [7], anti-allergic as well as anti-cancer properties [8]. Daidzein and genistein are the two main and most well characterized isoflavones present in soybean, able to inhibit transcription factors

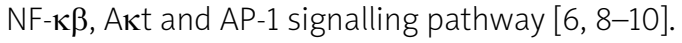

Extensive studies in the field of dermatology and cosmetology have demonstrated that phytoestrogens are effective in reducing skin ageing induced by oestrogen deprivation as they increase hyaluronic acid concentration [11], content and quality of collagen [12], and stimulate synthesis of the extracellular matrix proteins [13].

Recent research on soy isoflavones' (SI) property reveals that the compounds can protect against UVinduced oxidative damage of the skin cells [14-16] by upregulation of the intracellular superoxide dismutase (SOD) [17] and catalase (CAT) activities etc. [14, 17, 18]. Gender differences in the skin tissue were observed in the distribution of $E R \alpha$ and $E R \beta$ in the epidermis and se-

Address for correspondence: Joanna Kruk, Faculty of Physical Culture and Health, University of Szczecin, 40 b/6 Piastów St, 71-065 Szczecin, Poland, phone: +48 91444 34 35, e-mail: joanna.kruk@usz.edu.pl Received: 26.06.2018, accepted: 24.07.2018. 
baceous gland cells $[19,20]$. A number of studies have provided evidence that oestrogens, via ERs, can influence the epidermis and dermis thickness, skin elasticity, skin moisture and firmness, pigmentation, vascularity, function of the skin immune system, growth of hair, and wound healing [18, 21]. In addition, oestrogens may improve/maintain the skin barrier function and regeneration [19]. However, to date, studies dealing with the oestrogen influence on the skin tissue were mainly performed in female animals or in postmenopausal women seeking alternatives to oestrogen treatment, while their effect on male rats' skin was rarely studied, and the findings are sparse and equivocal.

\section{Aim}

The current study is different from previously published articles as we aimed to investigate daidzein and genistein administration from prenatal life until sexual maturity on morphology and the cellular redox status in the skin of male rats.

\section{Material and methods}

Sexually mature 3-month-old female Wistar rats were kept for a week in a cage with sexually mature males $(2: 1)$ under standard conditions in terms of lighting (12L : 12D) and nutrition. After a week, they were separated from the males, and each female was placed in a separate cage. Pregnant females were randomly divided into 3 groups (4 rats per group: control (C) and experimental groups (S2 and S20)). Females of the experimental groups received per os soy isoflavones (SI) daidzein and genistein mixture (Meno Stop - HASCO Lek, Poland). Based on the content of these compounds in soy human diet [22], we chose two dose groups for SI treatment: $2 \mathrm{mg} / \mathrm{kg}$ body weight/day (bw/day) (low doses, S2 group) and $20 \mathrm{mg} / \mathrm{kg}$ bw/day (high doses, 520 group). Rats were treated with SI from the first day of the experiment until delivery. Isoflavones were given once a day for 5 days/week to each rat mixed with a regular rat chow. The females and young males of the control group $(n=8)$ received regular rat chow from the feed store. Young males of experimental groups ( $n=8$ rats per group) were continuously treated with the same doses of SI until reaching the age of sexual maturity. Next, the rats were sacrificed under thiopental anaesthesia (120 mg/kg bw, i.p., Biochemie $\mathrm{GmbH}$, Austria). The study was performed according to the National Institute of Health Guidelines for the Care and Use of Laboratory Animals and the European Community Council Directive for Care and Use of Laboratory Animals and was approved by a local ethics committee (Committee on the Use and Care of Animals, approvals No. 25/2012, 26/2012, and No. BN-03/12).

\section{Morphometric analysis}

The skin samples were fixed in freshly prepared $4 \%$ paraformaldehyde ( $\mathrm{pH}$ 7.4). After fixation, the skin was embedded in paraffin wax, sectioned and stained with hematoxylin and eosin $(\mathrm{H}-\mathrm{E})$. The thickness of the epidermis and the diameter of collagen fibers in the dermis of rats' skin were measured using a Zeiss microscope equipped with a 20x objective lens. For each animal, 50 measurements were carried out. Additionally, in skin sections stained with $\mathrm{H}-\mathrm{E}$, the number of fibroblasts was counted using ocular $10 \times$ with a square reticle. The surface of each square was $1225 \mu^{2}{ }^{2}(35 \times 35 \mu \mathrm{m})$ under 40× objective lens. All morphometric measurements were performed with Axio Scope A1 (Zeiss) using AxioVision Rel. 4.6 Software.

\section{Biochemical study}

To determine the activity of SOD, CAT, glutathione peroxidase (GPX), glutathione reductase (GR), and concentrations of glutathione (GSH) and lipid peroxides (LPO), the tissue homogenates were prepared as follows: equal volumes of skin samples ( $0.5 \mathrm{~g}$ ) were placed in phosphate buffered saline, $\mathrm{pH}=7.4$, frozen in liquid nitrogen and underwent homogenization using a hammer mill. The suitable buffer (5-10 ml/g tissue) was added, and then tissues underwent homogenization using a CAT X120 homogeniser (Germany). The homogenates were centrifuged for $10 \mathrm{~min}$ at $12,000 \mathrm{G}$ at $4^{\circ} \mathrm{C}$. The supernatants were used to determine the activities of SOD, CAT, GPx, GR, and the levels of GSH and LPO were measured using suitable Assay Kits (Cayman Chemical Company) following the manufacturer's instructions. The absorbance was measured at suitable wavelengths with a usage of plate reader Asys UVM 340 (Asys Hitech GmbH, Austria).

Results of enzymes activity were converted into the total protein content, which was determined using a commercial BCA Protein Assay Kit (Thermo Scientific Pierce, USA). An absorbance was measured at $562 \mathrm{~nm}$.

\section{Statistical analysis}

The data were analysed statistically using Statistica PL 6.1 software. The data are presented as mean \pm SE of the mean. The distribution of results for individual variables was obtained by the Shapiro-Wilk test. The nonparametric Mann-Whitney U-test was used to determine significant differences between means. $P$-value of $<0.05$ was regarded as significant.

\section{Results}

The thickness of the epidermis in the skin of the control and experimental male rats' groups was measured in cross sections of the organ. As shown in Figure $1 \mathrm{~A}$, the epidermis thickness in the skin of rats exposed to SI was significantly greater $(p<0.001)$ than the control group. The values for the median thickness of epidermis 


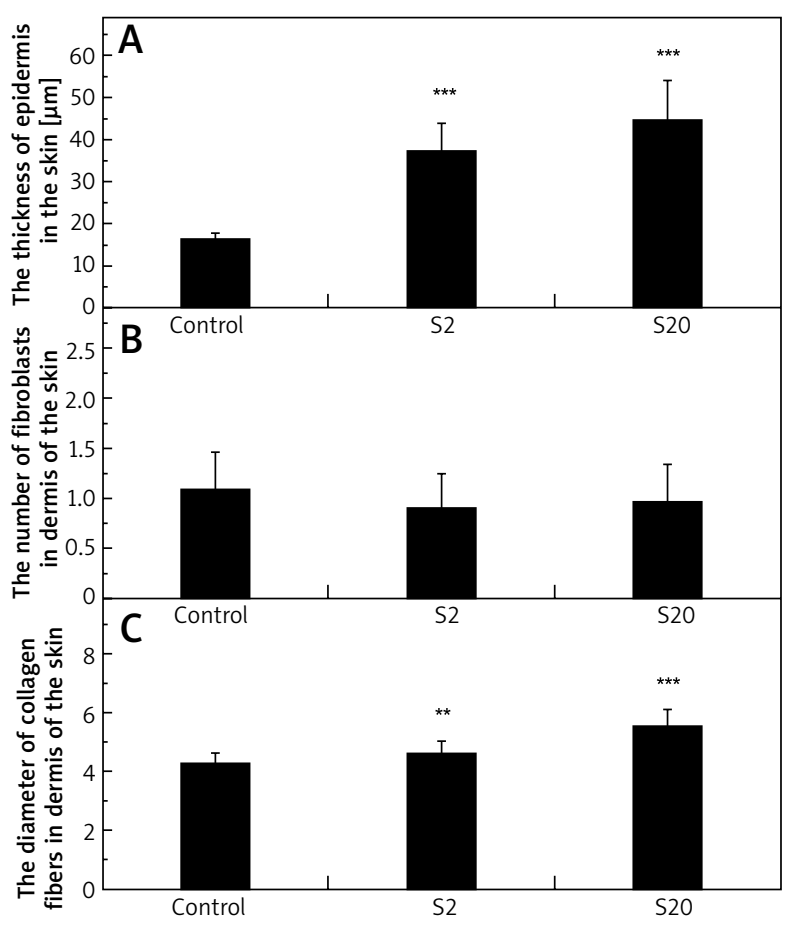

Figure 1. The thickness of epidermis in the skin (A), the number of fibroblasts in dermis of the skin (B), and diameter of collagen fibers in dermis of the skin (C) of rats in the control group and rats exposed to soy isoflavones in a dose of $2 \mathrm{mg} / \mathrm{kg}$ bw/day (S2) or $20 \mathrm{mg} / \mathrm{kg}$ bw/day (S20). Results are given as mean \pm SE of 8 rats in each group. Values denoted as $\left({ }^{* \star}\right)$ and $\left({ }^{* * *}\right)$ are significantly different from the control value at $p \leq 0.01$ and $p \leq 0.001$, respectively

and lower and upper quartiles for the control, 52 and S20 $(\mu \mathrm{m})$ groups were: $16.95,13.91--18.67 ; 32.70,24.50$ 42.74; 37.37, 28.90-50.63, respectively.

No statistically significant differences were noted in the number of fibroblasts in the dermis of the skin between the control group and the groups of rats exposed to SI (Figure 1 B). The values for median, and lower and higher quartiles of the fibroblasts number for the control, S2 and S20 groups were: 1, 0-2; 1, 0-1; 1, 0-2, respectively.

A statistically significant increase in the thickness of collagen fibers was observed in the dermis of rats exposed to SI ( $p<0.01$ for S2 group and $p<0.001$ for S20 group) compared with the control group (Figure $1 \mathrm{C}$ ). Additionally, a significant difference in collagen fibers diameter in the dermis was noted between S2 and S20 groups, the diameter being higher in the S20 group. The values for the median diameter of collagen fibers and lower and higher quartiles for the control, S2 and, S20 groups ( $\mu \mathrm{m})$ were: 4.22, 3.48-5.02; 4.43, 3.89-5.28; 5.64, 4.27-6.66, respectively.

\section{Biochemical studies of redox balance in the skin}

In the skin homogenates of the rats treated with a higher SI dose (group S20), LPO level was decreased compared

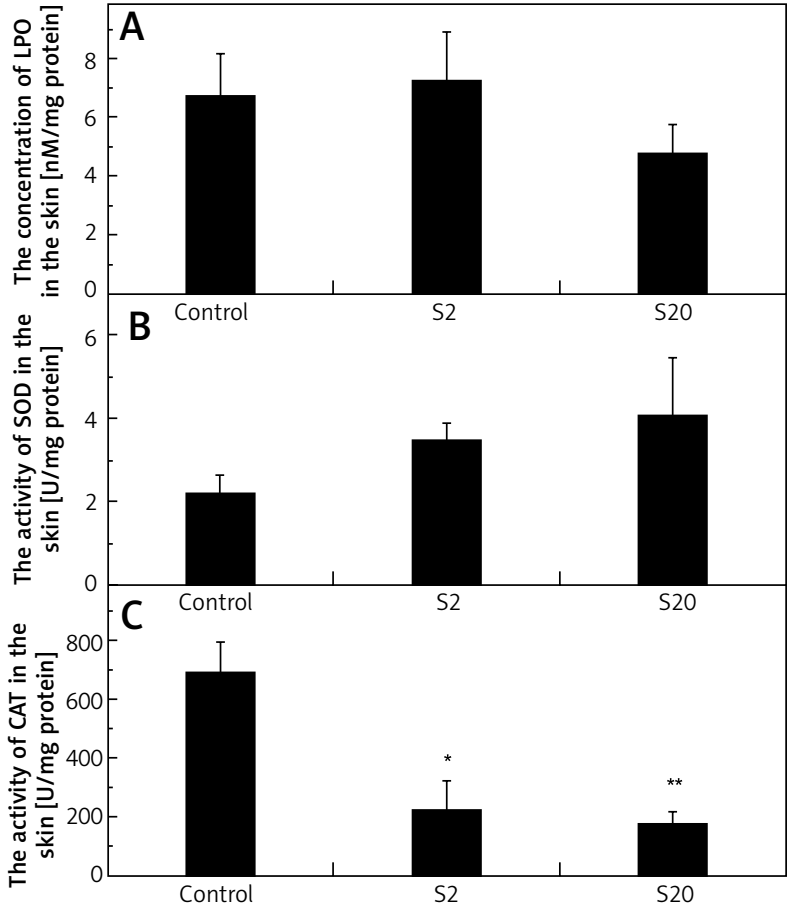

Figure 2. Concentrations of lipid peroxides (LPO) (A), the activities of superoxide dismutase (SOD) (B), and catalase (CAT) (C) in the skin homogenates of the control group and rats exposed to soy isoflavones in a dose of $2 \mathrm{mg} / \mathrm{kg}$ bw/ day (S2) or $20 \mathrm{mg} / \mathrm{kg}$ bw/day (S20). Results are given as mean \pm SE of 8 rats in each group. Values denoted as $\left(^{*}\right)$ and $\left({ }^{* *}\right)$ are significantly different from the control group value at $p \leq 0.05$ and $p \leq 0.01$, respectively

with the control group; however, the difference was not statistically significant (Figure 2 A). The values for the median LPO level and lower and higher quartiles for the control, S2 and S20 groups (nM/mg protein) were: 6.35, 3.78-9.67; 4.73, 4.49-9.23; 4.30, 2.78-5.04, respectively.

No significant effect of SI treatment was found in SOD activity, however we found a higher enzyme activity in the treated groups compared with the control group (Figure 2 B). Values for median SOD activity and lower and higher quartiles for the control, S2 and S20 groups (U/mg proteins) were: 2.50, 1.26-3.13; 3.43, 2.21-4.78; 3.92, 2.18-6.75, respectively.

The activity of CAT was significantly lower in the skin of rats exposed to SI ( $p<0.05$ in S2 group and $p<0.01$ in S20 group) than the control group (Figure $2 \mathrm{C}$ ). The values for median CAT activity and lower and higher quartiles for the control, S2 and S20 groups (U/mg proteins) were: 706.42, 437.86-943.60; 152.57, 116.56-247.12, 110.43, 41.72-234.38, respectively.

No statistically significant differences were observed in GPx activity in the skin between the control group and groups of rats treated with SI (Figure $3 \mathrm{~A}$ ). The values for median enzyme activity and lower and higher quartiles for 
the control, S2 and S20 groups (U/mg proteins) were: 8.82, 5.98-10.84; 10.0, 3.96-14.30; 7.94, 4.11-10.18, respectively.

There were no statistically significant differences in GR activity between the skin of the control group and the group exposed to a low dose (2 mg/kg bw/day) of SI. In the skin of rats exposed to a dose of $20 \mathrm{mg} / \mathrm{kg}$ bw/day, GR activity was slightly higher compared to the control group, however the difference was not statistically significant (Figure 3 B). The values for median of GR activity and lower and higher quartiles for the control, S2 and S20 groups (U/mg proteins) were: 3.58, 2.25-9.21; 4.18, 2.41-9.38; 7.83, 4.19-12.00, respectively.

The levels of GSH in the skin homogenates of rats treated with SI at $2 \mathrm{mg} / \mathrm{kg}$ bw/day (S2) or $20 \mathrm{mg} / \mathrm{kg}$ bw/ day (S20) were slightly increased, but they were not statistically different compared to the control level (Figure $3 \mathrm{C})$. The values for median of GSH concentration in the skin homogenates and lower and higher quartiles for the control, S2 and S20 groups ( $\mu \mathrm{M} / \mathrm{mg}$ protein) were: 0.04 , 0.03-0.06; 0.05, 0.04-0.07; 0.05, 0.05-0.06, respectively.

\section{Discussion}

The present study was undertaken to assess the possible beneficial effect of genistein and daidzein mixture on male rats' skin parameters, such as thickness of the entire epidermis, diameter of collagen fibers, number of fibroblasts, and levels of antioxidant enzymes. To our knowledge, this is the first study to investigate the effects of SI on the skin of male rats exposed during foetal and postnatal periods until sexual maturity. The effect of phytoestrogens as an alternative to oestrogen treatment is well documented in postmenopausal women, but no clearly supported evidence was found in men [1, 23]. The oestrogen treatment in postmenopausal women increases collagen content, thickness of the epidermis and dermis, elasticity, wound healing, vascularization, and the proper functioning the skin's immune system [18, 21].

In the present experiment, morphometric analysis showed a significant increase in the thickness of the entire skin epidermis in male rats treated with SI. This observation is in line with the findings of a previous study which found the normal epidermal thickness restored, improved vascularization and skin elasticity in female rats after ovariectomy treated with SI [15]. Likewise, the use of concentrated SI by postmenopausal women resulted in an increased number of epidermal layers as well as in normalization of keratinization [23].

We hypothesized several mechanisms for SI benefit, first through upregulation of ER followed by the cell division of the male epidermis, which led to an increase in its thickness. The second mechanism of SI effect on epidermal regeneration is possible via oestrogen-independent pathway. Genistein can accelerate keratinocyte migration and reepithelization through the influence on insulin growth factor-1 (IGF-1) receptor [24]. The third suggested

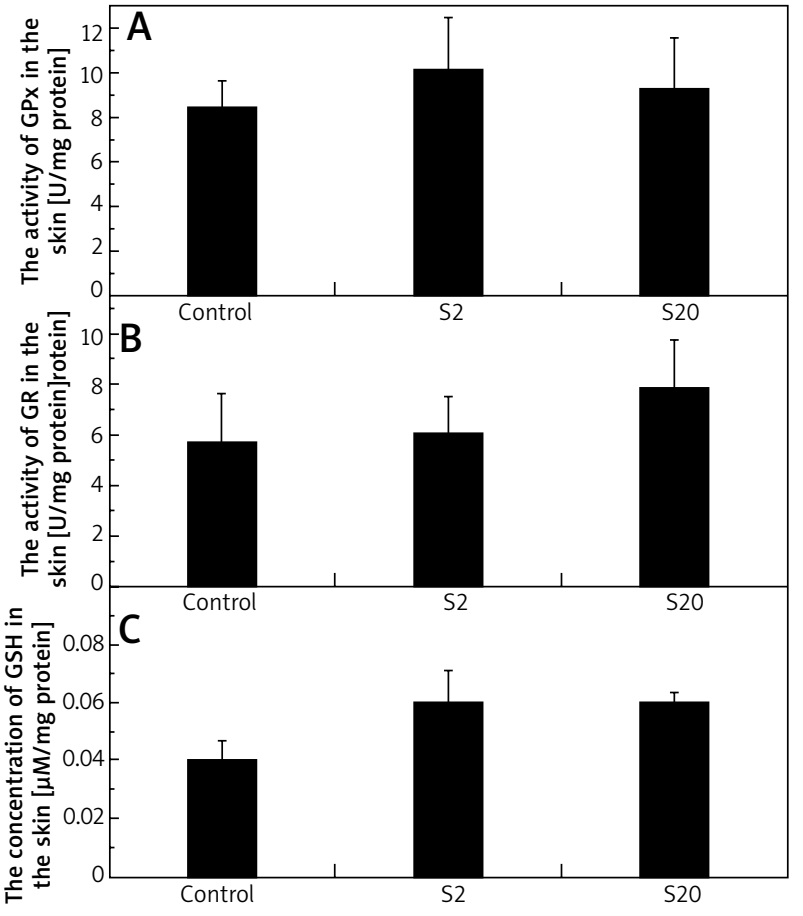

Figure 3. The activities of glutathione peroxidase (GPx) (A), glutathione reductase (GR) (B) and the concentration of glutathione (GSH) (C) in the skin homogenates of the control rats and rats exposed to soy isoflavones in a dose of $2 \mathrm{mg} / \mathrm{kg}$ bw/day (S2) or $20 \mathrm{mg} / \mathrm{kg}$ bw/day (S20). Results are reported as mean $\pm \mathrm{SE}$. $N=8$ rats/group

mechanism is linked with the anti-inflammatory properties of genistein. Previous studies demonstrated the effect of genistein on serine/threonine specific kinases, e.g. mitogen-activated protein kinases (MAPK), protein kinase A (PKA) as well as on expression of genes encoding GPx and Cu- dependent SOD [8]. Further, the increased thickness of the skin epidermis may result in the improved epidermal barrier function and effectiveness of protective functions of the male rats' skin. This ability of $\mathrm{SI}$ is important, considering that the epidermal barrier is less effective in males than in females [25]; this may offer new clues in this aspect.

Genistein and daidzein had no effect on the number of fibroblasts in the connective tissue of rats' dermis. So far, there is a limited research dealing with their effect on the number of fibroblasts in the skin. Recent studies have shown that phytoestrogens can exert a beneficial effect on collagen synthesis [2]. In vitro studies on human dermal fibroblasts have demonstrated that plant isoflavones stimulate extracellular matrix components synthesis, increase expression of collagen (especially procollagen $1 \mathrm{~A} 1)$, elastin and fibrillin [26].

Histological evaluation showed that texture of collagen fibers (collagen type I) and reticulin (collagen type III) in the papillary and reticular layers of male rats dermis 
treated with SI were similar to those of the control group (data not shown). Moreover, we found significant thickening of collagen fibers type I in the skin of the rats treated with SI. Texture of elastic fibers in the papillary and reticular layers of the rats' dermis treated with SI were not changed, however the content of elastic fibers in the dermal layer was higher, especially in the $\mathrm{S} 20$ group. This result is in an agreement with several previous reports [4, 26, 27]. For example, Zhao et al. demonstrated that daidzein can positively affect the collagen synthesis and inhibit its degradation under in vivo and in vitro conditions [26]. The authors found that transforming growth factor $\beta 1$ (TGF- $\beta 1$ ) and Smad-dependent signalling pathways play an important role in daidzein-dependent collagen deposition.

Evidence has shown that low levels of genistein stimulate, but high concentrations inhibit collagen synthesis [4]. This dual effect may be due to several modes of action, such as modulation of oestrogen and IGF-1 receptor, inhibition of tyrosine kinase activity, ribosomal 56 activity, and activation of aminoacyl tRNA synthetase [27]. An increased expression of TGF- $\beta 1$, vascular endothelial growth factor (VEGF), extracellular matrix metalloproteinases 2 and 9 (MMP-2 and MMP-9), and tissue inhibitors of metalloproteinase 1 and 2 (TIMP-1 and TIMP-2) by genistein was found [28].

The present study demonstrates a non-significantly decreased level of LPO in the skin homogenates of the S20 group. Phytoestrogens contribution to the regulation of prooxidant/antioxidant equilibrium in the body depends on their dose being tissue/site specific $[2,28]$ Thus, the lack of the significance may result from relatively low doses of SI. This result is in agreement with previous evidence $[28,29]$. There is a suggestion that SI are ROS scavengers and they significantly up-regulate expression and potency of the antioxidant genes and enzymes [30].

As in other tissues, the antioxidant enzyme system including SOD, CAT, GPx, GR and non-enzymatic antioxidants, e.g. GSH, play an important role in ensuring optimal levels of reactive oxygen species/nitrogen species (ROS/RNS) in the skin. In the present study, an exposure of rats to $\mathrm{SI}$ had no statistically significant effect on SOD activity in the skin, however we observed a slight increase in the enzyme activity. We can assume that SI were able to remove ROS directly by serving as hydrogen atoms or electron donors and/or that superoxide anion radical was not formed at an increased concentration under our experimental conditions [31, 32].

We observed that the exposure of male rats to SI resulted in a significant reduction in CAT activity in the skin homogenates. CAT usually works as an antioxidant when the $\mathrm{H}_{2} \mathrm{O}_{2}$ level is high, what probably did not occur in our study, as the animals were not further exposed to any factors that could increase ROS concentrations. We maintain that significant reactivity of $\mathrm{SI}$ towards $\mathrm{H}_{2} \mathrm{O}_{2}$ [33] could be responsible for a decrease in CAT activity.
Consistent with the present result, the significant reduction of CAT activity was demonstrated in serum, without affecting the enzyme activity in human organs and erythrocytes of immature male rats treated with mixed phytoestrogens [34]. In turn, other researchers observed a negligible decrease in CAT activity in the epididymis after exposure of mature rats to phytoestrogens [35].

We also found that exposure of animals to SI, regardless of their dosage, did not affect GPx and GR activities. Similar results were reported by Park et al. for GPX activity in the brain, kidneys, spleen and serum of the rats exposed to phytoestrogens [36]. In contrast to our study, Abarikwu et al. demonstrated a slight decrease in GR activity in epididymal homogenates of male rats [35].

Previous studies reported different effects of phytoestrogens on tissue levels of GSH $[35,37]$. For example, Abarikwu et al. found a lower GSH concentration in male rats' epididymal receiving phytoestrogens [35]. In contrast, our findings did not confirm the SI impact on the GSH concentration in male skin. Similarly, Chen et al. failed to confirm the effect of low concentrations of genistein on GSH content in myocytes culture [37].

A potential explanation for the inconsistent results among mentioned studies may originate from the multidirectional effects of isoflavones on the oxidant and antioxidant balance depending on their type, dose, exposure time, age, target tissue, and differences in the individual absorption and/or metabolism rate. Evidence shows that some isoflavones undergo oxidation forming ROS [2, 37]. In addition, the dichotomy (estrogenic and anti-estrogenic effects) of ER modulating by SI and molecular mechanisms of their action in each tissue which are dependent on the ER composition may explain observed conflicting findings [38].

Our results reveal that relatively low doses of daidzein and genistein in the male rats diet had a beneficial effect on the synthesis of collagen fibers and elastic fibers or inhibited their degradation process. Known high efficacy of the topical use of phytoestrogens in women and our findings allow to suggest that use of genistein and daidzein could improve also the quality of men skin components as well as slow down skin aging. The men's skin is more sensitive to UV radiation than that of women, what leads to its greater damage [39] so it requires special protection. Taking into account that genistein and other isoflavones or their metabolites applied locally on the skin of animals and women exhibited the protective effect on the skin ageing and neoplasm formation [1, 15] and our promising findings, we may conclude that the use of phytoestrogens to prevent males' skin from UV damage and ageing should be taken into consideration.

\section{Conclusions}

Morphometric analysis showed significant increases in the thickness of the skin epidermis, collagen type I fibers 
in the dermis, and in the amount of elastic fibers of male rats treated with the daidzein and genistein mixture. The examined isoflavones showed a tendency to extinguish lipid peroxidation and significantly lowered the activity of catalase in the rats' skin. It is very likely that the positive effects of the genistein - daidzein combination on the skin morphology of male rats could be linked with the selective activation of ER $\beta$. The compounds might work not only by their direct antioxidant action, but they also were able to influence the antioxidant enzyme system. Thus, our findings provide further support for the contribution of phytoestrogens to defence mechanisms against intracellular oxidative stress. However, the specific mechanism of their interactions in males requires further studies to determine the optimal dose, duration of use, and route of administration to achieve the best possible protection against skin ageing without side effects.

\section{Conflict of interest}

The authors declare no conflict of interest.

\section{References}

1. Shu YY, Maibach HI. Estrogen and skin: therapeutic options. Am J Clin Dermatol 2011; 12: 297-311.

2. Sirotkin AV, Harrath AH. Phytoestrogens and their effects. Eur J Pharmacol 2014; 741: 230-6.

3. Paterni I, Granchi C, Katzenellenbogen JA, Minutolo F. Estrogen receptors alpha (ERalpha) and beta (ERbeta): subtype-selective ligands and clinical potential. Steroids 2014; 90: 13-29.

4. Rietjens IM, Sotoca AM, Vervoort J, Louisse J. Mechanisms underlying the dualistic mode of action of major soy isoflavones in relation to cell proliferation and cancer risks. Mol Nutr Food Res 2013; 57: 100-13.

5. Evsen MS, Ozler A, Gocmez C, et al. Effects of estrogen, estrogen/progesterone combination and genistein treatments on oxidant/antioxidant status in the brain of ovariectomized rats. Eur Rev Med Pharmacol Sci 2013; 17: 1869-73.

6. Georgetti SR, Casagrande R, Vicentini FT, et al. Protective effect of fermented soybean dried extracts against TPA-induced oxidative stress in hairless mice skin. Biomed Res Int 2013; 2013: 340626.

7. Jia Z, Babu PV, Si H, et al. Genistein inhibits TNF-alpha-induced endothelial inflammation through the protein kinase pathway $A$ and improves vascular inflammation in C57BL/6 mice. Int J Cardiol 2013; 168: 2637-45.

8. Mahmud AM, Yang W, Bosland MC. Soy isoflavones and prostate cancer: a review of molecular mechanisms. J Steroid Biochem Mol Biol 2014; 140: 116-32.

9. Bakhtiari M, Panahi Y, Ameli J, Darvishi B. Protective effects of flavonoids against Alzheimer's disease-related neural dysfunctions. Biomed Pharmacother 2017; 93: 218-29.

10. Sobhy MMK, Mahmoud SS, El-Sayed SH, et al. Impact of treatment with a protein tyrosine kinase inhibitor (Genistein) on acute and chronic experimental Schistosoma mansoni infection. Exp Parasitol 2018; 185: 115-23.

11. Patriarca MT, Barbosa de Moraes AR, Nader HB, et al. Hyaluronic acid concentration in postmenopausal facial skin after topical estradiol and genistein treatment: a doubleblind, randomized clinical trial of efficacy. Menopause 2013; 20: 336-41.
12. Chua LS, Lee SY, Abdullah N, Sarmidi MR. Review on Labisia pumila (Kacip Fatimah): bioactive phytochemicals and skin collagen synthesis promoting herb. Fitoterapia 2012; 83: 1322-35.

13. Gopaul R, Knaggs HE, Lephart ED. Biochemical investigation and gene analysis of equol: a plant and soy-derived isoflavonoid with anti aging and antioxidant properties with potential human skin applications. Biofactors 2012; 38: 44-52.

14. Chiu TM, Huang CC, Lin TJ, et al. In vitro and in vivo antiphotoaging effects of an isoflavone extract from soybean cake. J Ethnopharmacol 2009; 126: 108-13.

15. Lim TG, Kim JE, Lee SY, et al. The daidzein metabolite, $6,7,4^{\prime}$-Trihydroxyisoflavone, is a novel inhibitor of PKCalpha in suppressing solar UV-induced matrix metalloproteinase 1. Int J Mol Sci 2014; 15: 21419-32.

16. Terra VA, Souza-Neto FP, Frade MA, et al. Genistein prevents ultraviolet $B$ radiation-induced nitrosative skin injury and promotes cell proliferation. J Photochem Photobiol B 2015; 144: 20-7.

17. Wang YN, Wu W, Chen HC, Fang H. Genistein protects against UVB-induced senescence-like characteristics in human dermal fibroblast by p66Shc down-regulation. J Dermatol Sci 2010; 58: 19-27.

18. Thornton MJ. Estrogens and aging skin. Dermatoendocrinology 2013; 5: 264-70.

19. Dao H Jr, Kazin RA. Gender differences in skin: a review of the literature. Gend Med 2007; 4: 308-28.

20. Lai JJ, Chang P, Lai KP, et al. The role of androgen and androgen receptor in skin-related disorders. Arch Dermatol Res 2012; 304: 499-510.

21. Campbell L, Emmerson E, Davies F, et al. Estrogen promotes cutaneous wound healing via estrogen receptor beta independent of its anti-inflammatory activities. J Exp Med 2010; 207: 1825-33.

22. Higdon J. Soy isoflavone. Linus Pauling Inst., Oregon State Univ. Available: http: http:///pi.oregonstate.edu/infocenter/ phytochemicals/soyiso/. Assesed 18 January 2014.

23. Accorsi-Neto A, Haidar M, Simões M, et al. Effects of isoflavones on the skin of postmenopausal women: a pilot study. Clinics 2009; 64: 505-10.

24. Emmerson E, Hardman MJ. The role of estrogen deficiency in skin ageing and wound healing. Biogerontology 2012; 13: 3-20.

25. Mizukoshi K, Akamatsu $\mathrm{H}$. The investigation of the skin characteristics of males focusing on gender differences, skin perception, and skin care habits. Skin Res Technol 2013; 19: 91-9.

26. Zhao D, Shi Y, Dang Y, et al. Daidzein stimulates collagen synthesis by activating the TGF-beta/smad signal pathway. Australas J Dermatol 2015; 56: e7-e14.

27. Sienkiewicz P, Surazyński A, Pałka J, Miltyk W. Nutritional concentration of genistein protects human dermal fibroblasts from oxidative stress-induced collagen biosynthesis inhibition through IGF-I receptor-mediated signaling. Acta Pol Pharm 2008; 65: 203-11.

28. Patel RP, Boersma BJ, Crawford JH, et al. Antioxidant mechanisms of isoflavones in lipid systems: paradoxical effects of peroxyl radical scavenging. Free Radic Biol Med 2001; 31: 1570-81.

29. Roghani M, Vaez Mahdavi MR, Jalali-Nadoushan MR, et al. Chronic administration of daidzein, a soybean isoflavone, improves endothelial dysfunction and attenuates oxidative stress in streptozotocin-induced diabetic rats. Phytother Res 2013; 27: 112-7. 
30. Borrás C, Gambini J, Gómez-Cabrera MC, et al. Genistein, a soy isoflavone, up-regulates expression of antioxidant genes: involvement of estrogen receptors, ERK1/2, and NFkappaB. FASEB J 2006; 20: 2136-8.

31. Ahmed SM, Luo L, Namani A, et al. Nrf2 signaling pathway: Pivotal roles in inflammation. Biochim Biophys Acta 2017; 1863: 585-97.

32. Xu SZ, Zhong W, Ghavideldarestani M, et al. Multiple mechanisms of soy isoflavones against oxidative stress-induced endothelium injury. Free Radic Biol Med 2009; 47: 167-75.

33. Kładna A, Berczyński P, Kruk I, et al. Studies on the antioxidant properties of some phytoestrogens. Luminescence 2016; 31: 1201-6.

34. Barbosa AC, Lajolo FM, Genovese MI. Effect of free or protein-associated soy isoflavones on the antioxidant status in rats. J Sci Food Agric 2011; 91: 721-31.

35. Abarikwu SO, Otuechere CA, Ekor M, et al. Rutin ameliorates cyclophosphamide-induced reproductive toxicity in male rats. Toxicol Int 2012; 19: 207-14.

36. Park E, Lee SM, Jung IK, et al. Effects of genistein on earlystage cutaneous wound healing. Biochem Biophys Res Commun 2011; 410: 514-9.

37. Chen W, Lin YC, Ma XY, et al. High concentrations of genistein exhibit pro-oxidant effects in primary muscle cells through mechanisms involving 5-lipoxygenase-mediated production of reactive oxygen species. Food Chem Toxicol 2014; 67: 72-9.

38. Chen KL, Madak-Erogan Z. Estrogens and female liver health. Steoroids 2018; 133: 38-48.

39. Oblong JE. Comparison of the impact of environmental stress on male and female skin. Br J Dermatol 2012; 166 Suppl. 2: 41-4. 